\title{
Why Correcting the Literature with Errata and Retractions is Good Medical Practice?
}

\author{
Allahbadia Gautam N.
}

Published online: 19 November 2014

(C) Federation of Obstetric \& Gynecological Societies of India 2014

\begin{abstract}
About the Author

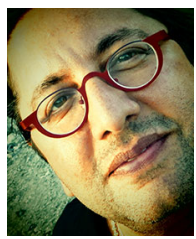

Gautam N. Allahbadia is the Editor-in-chief of the Journal of Obstetrics \& Gynecology of India as well as the IVF Lite (Journal of Minimal Stimulation IVF). He is the Medical Director of Rotunda-The Center for Human Reproduction, the world-renowned fertility clinic at Bandra, Mumbai, India — and also the New Hope IVF Clinic at Sharjah, UAE. He is a noted world authority on Ultrasound-guided Embryo Transfers and one of the pioneers in Third Party Reproduction in South-East Asia. Dr. Allahbadia was responsible for India's first trans-ethnic surrogate pregnancy involving a Chinese couple's baby delivered by an unrelated Indian surrogate mother. He has over 100 peer-reviewed publications to his credit and is on the Editorial Board of several International Journals. Throughout his career, Dr. Allahbadia has been instrumental in developing new fertility-enhancing protocols and propagating the use of Ultrasound in Embryo Transfer procedures. You can read more about his work at www.gautamallahbadia.com.
\end{abstract}

\section{Introduction}

The National Library of Medicine, USA (NLM) has a longstanding tradition of providing access to information in the biomedical literature [1]. One of the ways NLM assists users is to add subsequent notices of and/or linkages between citations for errata, retractions, partial retractions, corrected and republished articles, duplicate publications, comments (including author replies and expressions of concern), updated versions of articles, patient summaries, and republished (reprinted) articles indexed and available in NLM's online MEDLINE database. Users who search MEDLINE will be informed if they retrieved a citation for an article that has been corrected by an erratum notice,

Allahbadia G. N. ( $₫)$, Medical Director

Rotunda - The Center for Human Reproduction, Bandra,

Mumbai, India

e-mail: ivfwaladoc@gmail.com retracted or partially retracted, corrected and republished; been found to duplicate another article; generated a separately published commenting article; been updated by a subsequent article; and if a summary for patients has been published, or has been republished (reprinted) in another journal.

The incidence of literature corrections, whether in the form of errata or retractions, in medical research is low, but the numbers have been increasing [2-4]. Whether the increased incidence is the outcome of heightened awareness, easier detection and notice of corrections, and/or better publication practices, there is good reason to prevent and minimize the need for them. A variety of "authorized" agents can correct the literature. They can include authors, editors, publishers or journal owners, legal counsel, and representatives from the institution or organization where the work was produced (e.g., department heads, deans, or laboratory directors). The primary methods used for correcting the literature are errata and retractions, whereas 
expressions of concern are used to raise awareness to a possible problem in an article.

\section{Discussion}

The National Library of Medicine, USA (NLM) is the largest medical library in the world; it serves millions of medical researchers through MEDLINE and develops policies in response to issues that surface in the biomedical publishing world. Its Fact Sheet [1] outlines how it handles corrections to the literature. The ICMJE Uniform Requirements [5], which are endorsed by more than 1,000 journals, reflect the experiences of editors since 1978 and are updated regularly to address new issues in scientific publication. COPE, established in 1977 by medical journal editors and now with more than 7,000 members, provides retraction guidelines [6] and flowcharts [7] on best practices that include correcting the literature.

The information that these organizations provide offers the greater scientific community a useful framework for addressing issues related to correcting the literature.

\section{Errata}

Published changes or amendments to an earlier article, frequently referred to as corrections or corrigenda, are considered by NLM to be errata, regardless of the nature or origin of the error [1]. Errata identify a correction to a small, isolated portion of an otherwise reliable article. The NLM and other indexing organizations do not differentiate between errors that originate in the research process, such as errors in the methodology or analysis, and those that occurred in the publication process, such as typographical mistakes or printing errors.

In PubMed $^{\circledR}$, the erratum information typically appears as a text phrase such as

Erratum in: JAMA. 2004 Nov 24;292(20):2470.

NLM will only acknowledge errata when they are published in citable form: the erratum notice must appear on a numbered page in a subsequent issue of the journal in which the article was originally published.

\section{Retractions}

Retractions identify an article that was previously published and is now retracted through a formal issuance from the author, editor, publisher, or other authorized agent [1]. Retractions refer to an article in its entirety that is the result of a pervasive error, nonreproducible research, scientific misconduct, or duplicate publication. A "retraction in part" or a "partial retraction" is more significant than an erratum. A "retraction in part" is the result of an incorrect section or a particular portion of an article that is incorrect, leaving the majority of the information and the article's stated conclusions uncompromised by the removal of that portion of the content. If the notification in the journal is labeled as a retraction or withdrawal, then NLM will index it as a retraction. Of the two primary forms of literature corrections, retractions can be more difficult to attain. As indicated by the NLM, retractions are issued for the more serious literature corrections and to "expunge" the article or part of the article from the scientific record. Admitting to a significant error, careless practices, unethical handling of the work on the part of one or more authors, or that the article resulted from their misconduct is difficult for authors to admit. However, retractions are most easily published when all authors agree to the need for the retraction and to the language in which the retraction is described.

\section{Expressions of Concern}

The International Committee of Medical Journal Editors (ICMJE) introduced this indexing term [8]. The expression of concern is a publication notice that is generally made by an editor to draw attention to possible problems, but it does not go so far as to retract or correct an article. An editor who has a significant concern about the reliability of an article but not enough information to warrant a retraction until an institutional investigation is complete will sometimes use an expression of concern.

For a variety of reasons, correcting the literature is a critical part of medical research. First, it addresses unreliable information that is part of the public record. Second, corrections enable researchers to identify and use correct information, thereby saving time and resources. Third, corrections enhance a journal's reputation for taking a proactive role in publishing accurate information for its readership. The need for corrections may originate from honest error or from misconduct. Because of the vastness of the horizons of medical research, it is important to note that no single recognized method exists for addressing literature corrections.

Retractions of articles and citations to retracted work continue to be a cause for concern. In 1999, Budd et al. found 235 retracted publications in the biomedical literature for a 30-year period [9]. Nearly $40 \%$ were retracted because of misconduct. In 2011, the same group found 1,164 retracted articles in the 12-year period between 1997 and 2009 [4]. Of the 1,112 articles included for analysis, $55 \%$ were retracted for some type of misconduct or the other. While this number represents a small minority of the total number of publications in biomedicine, it is still substantial, and the impact of the retracted works can be 
significant. In PubMed, notifications of retractions are connected to the original publication; the retraction information appears when a retracted article is retrieved in a search. Citations to retracted articles should, theoretically, be minimal. Despite these notifications of retraction in PubMed and elsewhere, the articles continue to receive citations. The 1999 study found that the retracted articles received more than 2,000 post-retraction citations, with less than $8 \%$ of the citations acknowledging the retraction in any way [9].

Hauptman et al. sought to determine the frequency and significance of published errata in high impact factor journals [10]. This was a retrospective evaluation of errata reports for articles published in 20 English-language general medicine and cardiovascular journals (mean impact factor, 12.23; median, 5.52) over 18 months. Source of error, association between impact factor and errata occurrence, and a total of 557 articles were associated with errata reports (overall errata report occurrence 4.2 per 100 published original and review articles; mean of 2.4 errors per errata report). At least one major error that materially altered data interpretation was present in $24.2 \%$ of articles with errata. There was a strong association between impact factor and errata occurrence rate (rho $=0.869, P<0.001$ ). Across all errata, 51.0\% were not corrected, or the report did not specify whether a correction was made. The authors expounded that the reporting of errata across journals lacks uniformity and summarized that despite published criteria for authorship that mandate final approval of the manuscript by all authors, errors are frequent, including those that may materially change the interpretation of data. Increased vigilance by authors to prevent errata and consensus by journal editors on the format of reporting are warranted [10].

The reasons for retraction are important mainly because of the impact the original research work can have on subsequent research. For example, if a paper is retracted because tissue samples used in the research were contaminated (even if the contamination was unknown to the researchers), the said research may well be invalid. Any clinical treatments that might have been based on such work would have to be foregone until further research could be conducted.

The problems with misconduct can be even more profound. As Trikalinos et al. state, "Of the different types of misconduct, falsification is more egregious and typically affects the veracity of the report more than plagiarism, faked author or ethics approval, or duplication." [11] In the present wired world with rapid information dissemination in a matter of hours, the instances of misconduct are highly publicized and cause instant loss of faith and reputation in the minds of the general public as well as the scientific community.
The most infamous and publicized case of scientific misconduct in recent times was that of the South Korean researcher Hwang Woo Suk, who claimed to have made a major breakthrough in stem cell reproduction by cloning in the prominent journal Science; Hwang's work, which the South Korean government had committed US \$65 million for further research, was fabricated [12].

In an editorial in the Lancet, Horton urged researchers to avoid citing retracted works in order to create distance between them and the cases of misconduct [13]. Sox and Rennie go one step ahead and argue that "scientific misconduct is endemic, so the scientific community must prove its response." They recommend that all potential authors have to indicate that they have checked the National Library of Medicine databases, after verifying that that are not citing retracted work [14].

\section{Conclusion}

The scientific literature is a record of the search for truth. Publication of faked data diverts this search. The scientific community has a duty to warn people to ignore an article containing faked data and must try to prevent inadvertent citation of it. The scientific community accomplishes these tasks by publishing a retraction and linking it to the fraudulent article's citation in electronic indexes of the medical literature, such as PubMed.

The various entities responsible for educating physicians, accrediting institutions, privileging/credentialing, certifying, and licensing physicians currently have no common language or framework for fulfilling their responsibilities in a consistent, coordinated manner. A Guide to Good Medical Practice was explicitly intended for the first time to provide common language and a common framework for those organizations both in the UK [15] the USA [16], and the document is ideally applicable globally. This living document supports the development of a common view of professional responsibility among individual physicians. Publishing ethics should be added to this living document and correction of the literature should be part of Good Medical Practice (GMP) in the years to come to foster good medical research.

\section{References}

1. National Library of Medicine. Fact sheet: errata, retractions, partial retractions, corrected and republished articles, duplicate publications, comments (including author replies), updates, patient summaries, and republished (reprinted) articles policy for MEDLINE. Available at: http://www.nlm.nih.gov/pubs/factsheets/errata.html Accessed 30 Oct 2014. 
2. Furman JL, Jensen K, Murray F. Governing knowledge in the scientific community: exploring the role of retractions in biomedicine. Res Policy. 2012;41(2):276-90.

3. Wager E, Williams P. Why and how do journals retract articles? An analysis of Medline retractions 1988-2008. J Med Ethics. 2011;37(9):567-70.

4. Budd JM, Coble ZC, Anderson KM. Retracted publications in biomedicine: cause for concern. ACRL Conference, Philadelphia, PA: 2011. Available at:http://www.ala.org/acrl/sites/ala.org.acrl/ files/content/conferences/confsandpreconfs/national/2011/papers/ retracted_publicatio.pdf Accessed 30 Oct 2014.

5. International Committee of Medical Journal Editors (ICMJE). Uniform requirements for manuscripts submitted to biomedical journals. Available at http://www.icmje.org/ Accessed 30 Oct 2014.

6. Committee on Publication Ethics (COPE). Retraction guidelines. Available at: http://publicationethics.org/resources/guidelines/ Accessed 30 Oct 2014.

7. Committee on Publication Ethics (COPE). Flowcharts. Available at: http://publicationethics.org/resources/flowcharts Accessed 30 Oct 2014.

8. NISO/ALPSP Journal article versions (JAV) Technical Working Group. Journal article versions (JAV): Recommendations of the NISO/ALPSP JAV Technical Working Group. April 2008. NISO-RP-8-2008.
9. Budd JM, Sievert ME, Schultz TR, et al. Effects of article retraction on citation and practice in medicine. Bull Med Libr Assoc. 1999;87(4):437-43.

10. Hauptman PJ, Armbrecht ES, Chibnall JT, et al. Errata in medical publications. Am J Med. 2014;127(8):779.e1-85.e1. doi: 10.1016/j.amjmed.2014.03.012.

11. Trikalinos NA, Evangelou E, Ioannidis JP. Falsified papers in high-impact journals were slow to retract and indistinguishable from nonfraudulent papers. J Clin Epidemiol. 2008;61(5):464-70.

12. Kakuk P. The legacy of the Hwang Case: research misconduct in biosciences. Sci Eng Ethics. 2009;15(4):545-62.

13. Horton R. Scientific misconduct: exaggerated fear but still real and requiring a proportionate response. Lancet. 1999;354(9172): 7-8.

14. Sox HC, Rennie D. Research misconduct, retraction, and cleansing the medical literature: lessons from the Poehlman case. Ann Intern Med. 2006;144(8):609-613.

15. Good medical practice: general medical council, UK. http://www. gmc-uk.org/static/documents/content/Good_medical_practice__English_0914.pdf Accessed 30 Oct 2014.

16. Guide to good medical practice: USA Version 1.1, March 9, 2009. https://gmpusa.org/Docs/GoodMedicalPractice-USA-V1-1.pdf Accessed 30 Oct 2014 